\title{
Repercussões orais de drogas antineoplásicas: uma revisão de literatura
}

\section{Oral effects of anticancer drugs: a literature review}

\author{
Leila Guerreiro de Jesus* \\ Monise Cicchelli* \\ Gabriela Botelho Martins ${ }^{* *}$ \\ Manoela Carrera Cavalcante Pereira*** \\ Hayana Santos Lima ${ }^{* * * *}$ \\ Alena Ribeiro Alves Peixoto Medrado ${ }^{* * * * *}$
}

\section{Resumo}

Introdução: as neoplasias são responsáveis por grande parte das alterações que afetam os seus portadores e o diagnóstico precoce é fundamental para aumentar as chances de cura e garantir o bom prognóstico do paciente. Em diversos casos, a intervenção cirúrgica, a quimioterapia e a radioterapia são utilizadas para combater o câncer. O tratamento quimioterápico é muito comum, podendo ser realizado em monoterapia ou pela combinação de fármacos. De acordo com a farmacocinética do quimioterápico e as características do tratamento, como a frequência e a duração, diversos efeitos colaterais orais podem ser observados. Objetivo: relatar as possíveis repercussões orais decorrentes da utilização de fármacos antineoplásicos. Revisão de literatura: dentre os efeitos orais observados no organismo em consequência do tratamento quimioterápico, as alterações mais comuns ocorrem na mucosa bucal, interferindo na qualidade de vida do paciente, no tempo de internação e nos custos hospitalares. O efeito citotóxico dos quimioterápicos pode promover uma ação direta, que acarreta em descamação, ulceração, xerostomia e neurotoxicidade; uma ação indireta que proporciona o surgimento de hemorragias e infecções oportunistas, devido à imunossupressão provocada pelo medicamento. Considerações finais: são necessários estudos clínicos baseados em evidências científicas para a adequação de protocolos necessários à prevenção e para o controle das lesões orais decorrentes da toxicidade dos quimioterápicos.

Palavras-chave: Estomatite. Mucosa bucal. Quimioterapia.

\section{Introdução}

O câncer representa a segunda causa de morte por doenças no mundo ${ }^{1}$. O seu diagnóstico precoce, juntamente com tratamentos eficazes, melhora 0 prognóstico do paciente, aumenta as possibilidades de cura e prolonga a vida do indivíduo ${ }^{2}$. Os pacientes com doenças oncológicas ou onco-hematológicas, geralmente, são submetidos ao tratamento antineoplásico. As modalidades primárias básicas no tratamento do câncer envolvem cirurgia, quimioterapia e radioterapia. A quimioterapia é considerada a modalidade mais comum no tratamento antineoplásico, e sua ação se dá por meio da utilização de compostos químicos, chamados de quimioterápicos. Esses fármacos podem ser utilizados isoladamente ou de forma combinada para a realização da quimioterapia. De acordo com o tipo, a dosagem, a duração e a frequência, muitos efeitos adversos podem ser observados no organismo, como, por exemplo, severas alterações na mucosa bucal ${ }^{3}$.

É importante ressaltar que existem outros fatores que também irão determinar a gravidade dessas lesões, como a idade, o sexo, o estado nutricional, as alterações na produção salivar, o trauma local e o nível de higiene bucal, antes e durante a terapia do câncer $^{2-4}$. Uma vez que os agentes quimioterápicos atuam nas células em proliferação, sem distinguir

Graduanda em Odontologia pela Escola Bahiana de Medicina e Saúde Pública, Salvador, Bahia, Brasil.

Professora Adjunta do Instituto de Ciências da Saúde da Universidade Federal da Bahia, Salvador, Bahia, Brasil.

Professora Adjunta da Universidade do Estado da Bahia, Salvador, Bahia, Brasil.

Professora Adjunta da União Metropolitana de Educação e Cultura, Lauro de Freitas, Bahia, Brasil.

PhD. Professora Adjunta da Universidade Federal da Bahia e da Escola Bahiana de Medicina e Saúde Pública, Salvador, Bahia, Brasil. 
as células neoplásicas das normais, a mucosa bucal representa um dos alvos dessa terapia e pode apresentar-se edemaciada, inflamada e/ou ulcerada. Dessa forma, o surgimento dessas alterações pode comprometer a saúde sistêmica, prolongar o tempo de internação hospitalar, reduzir a sobrevida do paciente e contribuir para o aumento dos custos do tratamento, afetando diretamente a qualidade de vida dos pacientes ${ }^{5}$.

Relatos na literatura afirmam que cerca de $40 \%$ dos pacientes que se submetem ao tratamento do câncer serão acometidos por diferentes graus de mucosite oral ${ }^{3}$. Esse fato procede em virtude da alta sensibilidade dos tecidos bucais aos efeitos tóxicos dos quimioterápicos ${ }^{4}$. De acordo com o efeito citotóxico dos quimioterápicos, essas repercussões na cavidade oral podem ser decorrentes de uma estomatotoxicidade direta, que é caracterizada pelos efeitos não específicos nas células em divisão, provocando a redução da renovação das células do epitélio e, consequentemente, o aparecimento de descamação, ulceração, inflamação e atrofia, como ocorre na mucosite oral. Em algumas situações, os pacientes relatam secura na boca, o que promove um desconforto na alimentação e na fonação. Além disso, há um aumento no índice de cáries, independente da história odontológica pregressa do paciente. A xerostomia ocorre em virtude da concentração de agentes quimioterápicos na saliva e de sua potencialidade em induzir toxicidade ${ }^{6}$.

As lesões provenientes de estomatotoxicidade indireta ocorrem devido aos efeitos em células específicas. Tais efeitos podem provocar trombocitopenia ou granulocitopenia quando atingem a medula óssea, propiciando o surgimento de hemorragias e infecções oportunistas, respectivamente ${ }^{2}$. De fato, tem sido citado na literatura que os agentes antineoplásicos ocasionam uma supressão no sistema imune do paciente, oportunizando o surgimento de infecções ${ }^{6}$.

O objetivo desta revisão de literatura é abordar os efeitos colaterais de alguns agentes quimioterápicos utilizados para o tratamento de pacientes oncológicos, em especial suas possíveis repercussões na mucosa oral.

\section{Revisão de literatura}

A quimioterapia causa toxicidade aos tecidos normais e os efeitos adversos podem ser classificados em agudos e tardios, de acordo com o período em que ocorrem. Os efeitos adversos agudos ocorrem durante a quimioterapia e acometem tecidos com alta taxa de renovação celular, como a mucosa bucal. Já os efeitos adversos tardios podem apresentar-se meses ou anos após o tratamento, sendo observados em tecidos e órgãos de maior especificidade celular ${ }^{6}$.

\section{Agentes quimioterápicos}

\section{Cisplatina}

A cisplatina, denominada também de CDDP (cis-diaminodicloroplatina), é um quimioterápico classificado como agente alquilante. Esse é mais ativo na fase de repouso da célula e não age especificamente durante o ciclo celular, impedindo a divisão celular. Dentre os efeitos colaterais desse fármaco, situam-se a trombocitopenia e a leucopenia, que, de forma indireta, promove supressão do sistema imune do paciente.

Diante do quadro clínico de imunodepressão, a predisposição do organismo para o desenvolvimento de infecções oportunistas aumenta, a exemplo das infecções fúngicas, virais e bacterianas. Além desses aspectos, tem sido relatada uma íntima correlação do uso da cisplatina com o aparecimento da mucosite oral, sendo essa a mais comum das alterações bucais ${ }^{7}$. A referida droga pode estar associada a alterações na sensibilidade gustativa, a exemplo de ageusia, hipogeusia e disgeusia ${ }^{8}$.

Quimioterapia à base de cisplatina e radioquimioterapia concomitante são as primeiras escolhas para o tratamento do câncer na região de cabeça e pescoço em estágios avançados, em particular para o tratamento de tumores não ressecáveis ${ }^{9}$.

\section{Fluorouracil}

O fluorouracil ou 5-FU é um antineoplásico comumente utilizado no tratamento quimioterápico de tumores malignos do trato gastrintestinal ${ }^{10}$. Esse medicamento é considerado um antimetabólito que apresenta semelhança com as substâncias normais de uma célula. Sendo assim, as células absorvem esses antimetabólitos e, em consequência, tornam-se incapazes de se dividir.

Náuseas, vômitos e diarréia representam os efeitos colaterais mais frequentes do 5-FU em relação a outras manifestações tóxicas ${ }^{11}$. O 5-FU influencia no aparecimento de infecções, devido à imunossupressão também promovida por esse medicamento. A clássica manifestação oral dessa droga é caracterizada por eritema, dor, úlcera e edema.

Estudos realizados com animais em quimioterapia induzida por esse antineoplásico demonstraram que a mucosite oral se constitui no principal efeito deletério do fluorouracil ${ }^{12}$. A mucosite ocasionada pela quimioterapia com fluorouracil e cisplatina, por exemplo, está associada ao aumento no uso de antibióticos e analgésicos, devido à predisposição para infecções oportunistas e à toxicidade dolorosa, respectivamente $^{13}$. O fluorouracil também pode estar relacionado às alterações no paladar ${ }^{8}$. 


\section{Adriamicina}

A adriamicina ou doxorrubicina é classificada como um antibiótico antitumoral - a antraciclina. Geralmente, é prescrita para tumores com alta prevalência, inclusive para o tratamento daqueles que se desenvolvem em crianças. Este fármaco atua nas diferentes fases do ciclo celular e suas repercussões orais são vistas como efeitos diretos ou em consequência das repercusões da adriamicina nas glândulas salivares ${ }^{10}$. Dentre os efeitos adversos da adriamicina, a mucosite oral e a erupção cutânea apresentaram relevância estatística $(p=0,001$; $p=0,002$, respectivamente), em estudo realizado em pacientes com câncer de mama e ginecológico ${ }^{14}$. A severidade da mucosite quimioinduzida pode ser intensa e pode haver predisposição a outras infecções oportunistas graves. Adicionalmente, tem sido constatada alteração no paladar em pacientes que utilizam esse medicamento ${ }^{8}$.

\section{Ciclofosfamida}

A ciclofosfamida é um agente alquilante que age de forma semelhante à cisplatina e atua na fase de repouso da célula. É indicada, preferencialmente, para o tratamento de neoplasias malignas hematológicas, pré e pós-transplante de medula (BMT), pois promove tolerância enxerto-hospedeiro após o transplante de células-tronco hematopoéticas ${ }^{15}$. A ciclofosfamida é citada como um dos fármacos mais relacionados ao desenvolvimento da mucosite oral, juntamente com o fluorouracil e o metotrexato ${ }^{3}$.

A ciclofosfamida é uma droga imunossupressora potente, muito utilizada para o tratamento de doenças autoimunes, câncer e transplantes ${ }^{15}$. Em relação à supressão imunológica resultante da neutropenia provocada pela administração desse antineoplásico, infecções oportunistas são sempre relatadas associadas ao uso dessa droga. Existem relatos na literatura de que pacientes apresentaram ulcerações e hipossalivação como efeitos colaterais orais importantes da administração de ciclofosfamida e outros quimioterápicos associados, intensificando a necessidade de utilização de fármacos que controlem a neutropenia e a algesia provocadas pelas lesões orais $^{16}$.

\section{Metotrexato}

O metotrexato (MTX) é uma droga antineoplásica classificada como antimetabólito, estruturalmente análogo ao ácido fólico. Sua indicação é frequente em determinadas doenças autoimunes, devido ao seu efeito anti-inflamatório. Além disso, uma das suas principais indicações envolve o uso em pacientes com leucemia linfoblástica. Os antineoplásicos dessa classe atacam as células em fases específicas do ciclo celular e são semelhantes às substâncias produzidas pelo corpo, e, a partir da incorporação dessas, a divisão celular é interrompida ${ }^{17}$.

Desse modo, o metotrexato está diretamente relacionado à estomatotoxicidade, por meio da produção de citocinas inflamatórias que agem diretamente sobre o epitélio humano, com pico de concentração de $72 \mathrm{~h}$ após a sua administração, desempenhando um papel importante na iniciação e no desenvolvimento de efeitos colaterais que impactam na qualidade de vida dos pacientes ${ }^{18}$. Estudos revelam que os prejuízos são mais comuns na alimentação, na fala, no convívio social e no estado emocional, podendo interromper o tratamento quimioterápico ${ }^{6}$.

Os efeitos colaterais orais mais comuns da medicação são estomatite ulcerativa, glossite, gengivite e mucosite. Dentre essas alterações, a mucosite eritematosa, erosiva e ulcerativa é a consequência mais relatada do metotrexato, devido à sensibilidade das células epiteliais bucais e intestinais ${ }^{17}$, seguida da xerostomia, que também ocorre frequentemente, pois o metotrexato pode ser secretado pela saliva ${ }^{19}$, e da disgeusia, que, juntas, representam as três manifestações orais mais citadas ${ }^{6}$. Na literatura, tem sido analisada a sensação de secura bucal e alterações na consistência e no fluxo salivar de pacientes que se submetem ao tratamento com esse fármaco ${ }^{20}$. A mielossupressão, a leucopenia, a neutropenia e a trombocitopenia são observadas frequentemente em estudos e proporcionam o aparecimento de infecções oportunistas ${ }^{21}$.

\section{Mucosite oral}

A mucosite oral está comumente relacionada aos efeitos da quimioterapia e da radioterapia realizadas no tratamento do câncer². Essa complicação oral é caracterizada clinicamente por inflamação e ulceração de regiões da mucosa bucal e se inicia com o aparecimento de eritema, seguido de placas brancas descamativas, hemorragia, ulceração, edema e dor intensa ${ }^{6}$. Essa condição pode ser exacerbada por fatores locais, como trauma dentário ou colonização bacteriana ${ }^{4}$.

A sintomatologia dolorosa, dependendo do grau de classificação da mucosite, pode comprometer funções indispensáveis do paciente, como a fala e a ingestão de alimentos e líquidos, o que implica na necessidade de nutrição parenteral e no tratamento direcionado para as lesões orais, ocasionando um aumento dos custos e do tempo de internação ${ }^{2}$. Além desses aspectos, a dor poderá interferir na dificuldade de higienização, contribuir para o surgimento de infecções oportunistas, a exemplo do vírus do herpes simples (HSV), Candida albicans e bactérias Gram anaeróbicas negativas ${ }^{19}$, necessitando de medicação local ou sistêmica ${ }^{3}$.

De acordo com o sistema de graduação da Organização Mundial de Saúde, a mucosite oral pode ser classificada em cinco graus: grau 0 - corresponde 
à ausência de mucosite; grau I - apresenta úlcera indolor, eritema ou sensibilidade leve; grau II - caracteriza-se por eritema doloroso, edema ou úlceras que não interferem na alimentação do paciente; grau III - úlceras que interferem na ingestão de alimentos sólidos; grau IV - sintomas muito severos que interferem na ingestão de líquidos e requerem suporte enteral ou nutrição parenteral ${ }^{6}$.

O desenvolvimento biológico da mucosite pode ser classificado em cinco fases, apesar de ser considerado um processo dinâmico: iniciação (I), geração de sinais mensageiros (II), sinalização e amplificação (III), ulceração (IV) e cicatrização (V). A fase de iniciação ocorre imediatamente após a administração da quimioterapia, promovendo um dano direto ao DNA de algumas células do epitélio e da submucosa. Simultaneamente, ocorre a formação de espécies reativas de oxigênio (ROS), desencadeando uma série de eventos biológicos. Na fase seguinte, a quimioterapia e as ROS ativam uma série de fatores de transcrição. Dentre eles, o fator nuclear kappa B $(\mathrm{NF}-\mathrm{\kappa B})$ é considerado o mais importante, pois uma vez ativado, ocorre a super-regulação de inúmeros genes que codificam citocinas pró-inflamatórias e moléculas de adesão. Na terceira fase, as citocinas irão promover a injúria tecidual direta e o dano indireto por meio da amplificação/sinalização do NF$\kappa \mathrm{B}$, promovendo um feedback positivo com essas citocinas. A fase ulcerativa é a mais importante do ponto de vista clínico e sintomático. As úlceras são caracterizadas pela profundidade, confluência, presença de bactérias e de um infiltrado inflamatório crônico composto principalmente por macrófagos. $\mathrm{Na}$ fase final, sinais provenientes da matriz extracelular estimulam a migração de células epiteliais, promovendo a proliferação e a diferenciação dessas. As úlceras cicatrizam espontaneamente por intermédio de um processo dinâmico ${ }^{22}$.

O tratamento e a prevenção da mucosite oral devem ser realizados com um acompanhamento multidisciplinar do paciente, a fim de acarretar uma melhoria na qualidade de vida das pessoas que fazem uso de antineoplásicos. Ainda não existe um protocolo definido para o tratamento da mucosite oral e muitos tratamentos tradicionais são ineficazes devido à sua alta complexidade biológica. Dessa maneira, as medicações são utilizadas na tentativa de promover o alívio dos sintomas ${ }^{19}$. A utilização de bochechos com o chá de camomila, por exemplo, pode conferir alívio dos sintomas da mucosite oral. A administração da pilocarpina oral pode estimular o fluxo salivar e a produção de mucina. Adicionalmente, crioterapia, analgésicos e palifermina proporcionam conforto para o indivíduo que desenvolve essa condição oral e, de forma indireta, contribuem para a redução dos níveis de desnutrição e perda de peso $^{23-25}$, proporcionando um aumento da sobrevida do paciente.

Além disso, o uso de sulfato de magnésio por via intravenosa tem sido descrito na literatura e parece exercer um efeito positivo na redução do grau de inflamação decorrente da terapia contra o câncer, em especial, sobre a mucosite oral ${ }^{26}$. O laser de baixa potência tem se mostrado um método seguro, eficiente e bem tolerado para a prevenção e o tratamento das mucosites orais. Estudos recentes apontam efeitos benéficos dessa terapia, devido ao seu efeito analgésico, anti-inflamatório e biomodulador. Devido à diversidade de aparelhos disponíveis no mercado, com diferentes potências e comprimento de onda variável, ainda não existe um protocolo definido para sua utilização. Novos estudos precisam ser realizados na tentativa de se estabelecer um protocolo fixo de aplicação do laser, para que, dessa maneira, possa ser incorporado nas equipes oncológicas ${ }^{27}$.

Hoje, existem cuidados especiais específicos aos pacientes que fazem quimioterapia, e cabe aos cirurgiões-dentistas a avaliação, a prevenção e 0 tratamento das lesões decorrentes da terapia quimioterápica. Com isso, a estabilização das condições bucais antes do início da terapia oncológica e o acompanhamento pelo profissional de saúde durante o tratamento podem reduzir o risco de aparecimento de complicações bucais ${ }^{28}$.

\section{Discussão}

A literatura evidencia amplamente a correlação entre os tratamentos quimioterápicos e o desenvolvimento de lesões orais ${ }^{2,3,7,12}$. A magnitude desses efeitos depende de uma série de fatores relacionados ao tratamento, ao tumor e ao paciente. No estudo realizado por Hespanhol et al. ${ }^{3}$ (2010), ficou estabelecido que as complicações orais oriundas do tratamento quimioterápico representam as principais reações adversas, sendo as mais frequentes a mucosite, a xerostomia e as infecções bacterianas, viróticas ou fúngicas, as quais comprometem a qualidade de vida desses pacientes.

Os agentes quimioterápicos com maior risco ao desenvolvimento da mucosite oral são o 5 -FU, a cisplatina, a ciclofosfamida e o metotrexato. Essa condição depende não só do ciclo de quimioterapia proposto, mas, também, do número de ciclos, pois múltiplos ciclos podem promover um efeito cumulativo em pacientes com histórico prévio de mucosite. Além disso, o uso concomitante da terapia de radiação aumenta significativamente esse risco. Em pacientes submetidos à terapia antineoplásica com altas doses em associação com transplante alogênico, o risco de desenvolver essa condição está associado principalmente ao regime de condicionamento. Para esses pacientes, o risco aumenta quando o metotrexato é utilizado para prevenir a rejeição do enxerto agudo no hospedeiro ${ }^{29}$.

Em estudo realizado por Morón e Viera ${ }^{18}$ (2012), citocinas pró-inflamatórias foram avaliadas na saliva, em resposta imunológica à administração do 
MTX. Os resultados obtidos indicaram uma liberação significativa de citocinas envolvidas no processo inflamatório capaz de afetar o tecido epitelial e submucoso da cavidade oral. Esses achados se correlacionam com o princípio das cinco fases da mucosite estabelecido por Sonis, uma vez que o nível inflamatório se encontra elevado nos primeiros dias após a utilização da droga, coincidindo com as fases II e III da mucosite oral.

Inúmeras escalas são utilizadas para descrever a severidade da mucosite oral ${ }^{29}$. Um bom sistema de pontuação deve cumprir alguns critérios, como conteúdo, validade e confiabilidade. Tradicionalmente, o conteúdo e a validade são estabelecidos por meio de uma pesquisa literária relevante e da solicitação de opiniões e ideias de especialistas na área. A confiabilidade é válida quando se demonstra a reprodutibilidade do sistema de pontuação utilizado pela mesma pessoa e/ou por diferentes indivíduos durante um período de tempo definido ${ }^{19}$.

A lista dos sistemas de pontuação da mucosite oral mais comumente utilizados inclui o sistema de pontuação proposto pela Organização Mundial de Saúde, o Instituto National do Câncer, o Grupo Oncológico de Radioterapia e o Instituto Nacional de Câncer do Canadá6,19. Pode ser difícil a concepção de uma única escala apropriada em todas as situações clínicas da mucosite, dada a diversidade de tratamentos antineoplásicos disponíveis e as suas resultantes toxicidades. No entanto, com o advento de novas formas de tratamento e a maior ênfase na avaliação da morbimortalidade relacionada ao tratamento, existe uma forte necessidade de aceitação universal de escalas para avaliar essa enfermidade ${ }^{19}$.

\section{Considerações finais}

O tratamento antineoplásico acarreta diversos efeitos colaterais, dentre os quais as manifestações orais decorrentes da estomatotoxicidade e da imunossupressão ${ }^{1}$. A mucosite oral, vista como a mais clássica das repercussões orais, além da xerostomia, é extremamente dolorosa e causa desconforto para o paciente, interferindo na fala e na ingestão de alimentos ${ }^{3}$. Dessa forma, pode modular o estado geral de saúde do paciente e aumentar o tempo de internação e os custos hospitalares.

A complexidade da manifestação oral determina a interrupção do tratamento oncológico, caso seja necessário, já que esse fator reduz a sobrevida do paciente e o predispõe a inúmeras infecções. Assim, o enfoque na atuação do cirurgião-dentista em equipes multidisciplinares oncológicas é indispensável, a fim de estabelecer uma boa qualidade de vida ao paciente em tratamento.

É necessária a realização de medidas de controle, prevenção e tratamento das lesões orais. Estudos clínicos que avaliem as manifestações orais mais prevalentes decorrentes da administração de qui- mioterápicos poderão também contribuir para uma melhor compreensão das alterações bucais que se desenvolvem nos pacientes oncológicos.

\section{Abstract}

Neoplasias are responsible for a great portion of changes that affect individuals with cancer, and early diagnosis is critical to increase the chances of cure and ensure good patient prognosis. In many cases, surgery, chemotherapy, and radiotherapy are used to fight cancer. Chemotherapy is a very common treatment and it may be performed as monotherapy or by the combination of drugs. According to the pharmacokinetics of anticancer drugs and aspects such as frequency and duration of treatment, several oral side effects may be observed. Objective: To report the potential oral repercussions derived from the use of anticancer drugs. Literature review: Among the oral effects observed in the body because of treatment, the most common changes occur in the oral mucosa, affecting the patient's quality of life, the length of hospital stay, and hospital costs. The cytotoxic effect of anticancer drugs may promote a direct action that results in flaking, ulceration, xerostomia, and neurotoxicity, and an indirect action that causes the appearance of bleeding and opportunistic infections due to immunosuppression caused by the drug. Final Considerations: Clinical studies based on scientific evidence are required for the adaptation of protocols directed to the prevention and control of oral lesions arising from the toxicity of anticancer drugs.

Keywords: Chemotherapy. Oral Mucosa. Stomatitis.

\section{Referências}

1. Belizário EJ. O próximo desafio reverter o câncer. Ciênc Hoje 2002; 184(31):50-7.

2. Martins ACM, Gaeti WP, Caçador NP. Complicações bucais da quimioterapia antineoplásica. Acta Sci 2002; 24(3):66370 .

3. Hespanhol FL, Tinoco BEM, Teixeira HGC, Falabella MEV, Assis NMSP. Manifestações bucais em pacientes submetidos à quimioterapia. Ciênc \& Saúde Colet 2010; 15(1):1085-94.

4. Raber-Durlacher JE, Elad S, Barasch A. Oral mucosits. Oral Oncol 2010; 46:452-6.

5. Brancher AP. Estomatotoxicidade da quimioterapia e seu impacto na qualidade de vida de pacientes do serviço de onco-hematologia do HU/UFSC [Monografia de Odontologia do Centro de Ciências da Saúde]. Santa Catarina: Universidade Federal de Santa Catarina; 2013.

6. Paiva CI, Zanatta FB, Flores DM, Pithan AS, Dotto GN, Chagas AM. Efeitos da quimioterapia na cavidade bucal. Disciplinarum Scientia 2004; 4(1):109-19.

7. Araújo SN, Luz MLBA, Almeida LHRB, Silva GRF, Moita Neto JM, Costa ACMMA. Pacientes oncológicos e a enfermagem: relação entre o grau de mucosite oral e a terapêutica implementar. J Res Fundam Care Online 2013; 5(4):386-95.

8. Amaral SM, Miranda AMMA, Pires FR. Reações medicamentosas na cavidade oral: aspectos relevantes na estomatologia. Rev Bras Odontol 2009; 66(1):41-53. 
9. Wang C, Liu XQ, Hou JS, Wang JN, Huang HZ. Molecular mechanisms of chemoresistance in oral cancer. Chin J Dent Res 2016; 19(1):25-33.

10. Volpato LER, Silva TC, Oliveira TM, Sakai VT, Machado MAAM. Mucosite bucal rádio e quimioinduzida. Rev Bras Otorrinolaringol 2007; 73(4):562-68.

11. Coninx P, Nasca S, Lebrun D, Panis X, Lucas P, Garbe E, et al. Sequential Trial of Initial Chemotherapy for Advanced Cancer of the Head and Neck. Cancer. [periódico on-line] 1988; [citado 2016 mar. 30]; 1; 62(9):188892. Disponível em URL: http://onlinelibrary.wiley.com/ doi/10.1002/1097-0142(19881101)62:9\%3C1888::AID-CNCR2820620903\%3E3.0.CO;2-M/pdf.

12. Lopez TC, Martins MD, Pavesi VC, Ferreira LS, Bussadori SK, Moreira MS, et al. Effect of laser phototherapy in the prevention and treatment of chemoinduced mucositis in hamsters. Braz Oral Res 2013; 27(4):342-8.

13. Limaye SA, Haddad RI, Cilli F, Sonis ST, Colevas AD, Brennan MT, et al. Phase 1b, multicenter, single blinded, placebo-controlled, sequential dose escalation study to assess the safety and tolerability of topically applied AG013 in subjects with locally advanced head and neck cancer receiving induction chemotherapy. Wiley Online Library 2013; 119(24):4268-76.

14. Boers-Sonderen MJ, van Herpen CM, van der Graaf WT, Desar IM, van der Logt MG, de Beer YM, et al. Correlation of toxicity and efficacy with pharmacokinetics (PK) of pegylated liposomal doxorubicin (PLD) $\left(\right.$ Caelyx $\left.^{\circledR}\right)$. Cancer Chem Pharm 2014; 74(3):457-63.

15. Xu X, Zhang X. Effects of cyclophosphamide on immune system and gut microbiota in mice. Microbiol Res 2015; 171:97-106.

16. Guerreiro MD, Swenson KK. Herpes simplex vírus-related oral mucositis in patients with lymphoma. Onc Nurs Forum 2014; 41(3):327-30.

17. Pedrazas CHS, Azevedo MNL, Torres SR. Oral events related to low-dose methotrexate in rheumatoid arthritis patients. Braz Oral Res 2010; 24(3):368-73.

18. Morón A, Viera N. Metotrexate como inductor de mucositis oral y producción de citocinas proinflamatorias: estudio in vivo e in vitro. Universidad del Zulia, Maracaibo 2012; 1-62.

19. Pico JL, Avila-Gavarito A, Naccache P. Mucositis: Its Occurrence, Consequences, and Treatment in the Oncology Setting. The Oncologist 1998; 3:446-51.

20. Jensen SB, Mouridsen HT, Reibel J, Brunner N, Nauntofte B. Adjuvant chemotherapy in breast cancer patients induces temporary salivary gland hypofunction. Oral Oncol 2008; 44(2):162-73.

21. Levinsen M, Rosthoi S, Nygaard U, Heldrup J, Harila-Saari A, Jonsson OG, et al. Myelotoxicity after high-dose methotrexate in childhood acute leucemia is influenced by 6 -mercaptopurine dosing but not by intermediate thiopurine methyltransferase activity. Cancer Chemother Pharmacol 2015; 75(1):59-66

22. Sonis ST. A biological approach to mucosits. J Support Oncol 2004; 2:21-36.

23. Lee HO, Lee JJ. Nutritional intervention using nutrition care process in a malnourished patient with chemotherapy side effects. Clin Nutr Res 2015; 4:63-7.

24. Eilers J, Million R. Prevention and management of oral mucositis in patients with cancer. Seminars in Oncology Nursing 2007; 23(3):201-12.

25. Gondim FM, Gomes IP, Firmino F. Prevenção e tratamento da mucosite oral. Rev Enferm UERJ 2010; 18(1):67-74.

26. Lee S. Mineral derivatives in alleviating oral mucosistis during cancer therapy: a systematic review. Peer J 2015; $3(765)$.
27. Oton-Leite AF, Silva GBL, Morais MO, Silva TA, Leles CR, Valadares MC, et al. Effect of low-level laser therapy on chemoradiotherapy-induced oral mucositis and salivary inflammatory mediators in head and neck cancer patients. Lasers in Surg and Med 2015; 47:296-305.

28. Zimmermann C, Meurer MI, Grando LJ, Del Moral JAG, Rath IB, Tavares SS. Dental Treatment in Patients with Leukemia. Journal of Oncology 2015; 1-14. doi $10.11555 / 2015 / 571739$.

29. Villa A, Sonis ST. Mucosits: pathobiology and management. Curr Opin Oncol 2015; 27:159-64.

\section{Endereço para correspondência:}

Alena R. A. Peixoto Medrado

Avenida Silveira Martins, 3.386

Cabula

41150-100 Salvador, Bahia

Telefone: (71) 8838-0218

E-mail: alenamedrado@hotmail.com

Recebido: 25/05/15. Aceito: 12/05/16. 